\title{
Variable Delay With Directly-Modulated R-SOA and Optical Filters for Adaptive Antenna Radio-Fiber Access
}

Prince, Kamau; Presi, Marco; Chiuchiarelli, Andrea; Cerutti, Isabella; Contestabile, Giampiero; Tafur Monroy, Idelfonso; Ciaramella, Ernesto Ciaramella

Published in:

Journal of Lightwave Technology

Link to article, DOI:

10.1109/JLT.2009.2026491

Publication date:

2009

Document Version

Publisher's PDF, also known as Version of record

Link back to DTU Orbit

Citation (APA):

Prince, K., Presi, M., Chiuchiarelli, A., Cerutti, I., Contestabile, G., Tafur Monroy, I., \& Ciaramella, E. C. (2009). Variable Delay With Directly-Modulated R-SOA and Optical Filters for Adaptive Antenna Radio-Fiber Access. Journal of Lightwave Technology, 27(22), 5056-5064. https://doi.org/10.1109/JLT.2009.2026491

\section{General rights}

Copyright and moral rights for the publications made accessible in the public portal are retained by the authors and/or other copyright owners and it is a condition of accessing publications that users recognise and abide by the legal requirements associated with these rights.

- Users may download and print one copy of any publication from the public portal for the purpose of private study or research.

- You may not further distribute the material or use it for any profit-making activity or commercial gain

- You may freely distribute the URL identifying the publication in the public portal 


\title{
Variable Delay With Directly-Modulated R-SOA and Optical Filters for Adaptive Antenna Radio-Fiber Access
}

\author{
Kamau Prince, Marco Presi, Andrea Chiuchiarelli, Isabella Cerutti, Giampiero Contestabile, \\ Idelfonso Tafur Monroy, and Ernesto Ciaramella, Member, IEEE
}

\begin{abstract}
We present an all-optical adaptive-antenna radio over fiber transport system that uses proven, commercially-available components to effectively deliver standard-compliant optical signaling to adaptive multiantenna arrays for current and emerging radio technology implementations. The system is based on a directly-modulated reflective semiconductor amplifier (R-SOA) and exploits the interplay between transmission-line dispersion and tunable optical filtering to achieve flexible true time delay, with $2 \pi$ beam steering at the different antennas. The system was characterized, then successfully tested with two types of signals defined in IEEE 802.16 (WiMAX) standard for wireless networks: a 90 Mbps single-carrier signal (64-QAM at $2.4 \mathrm{GHz}$ ) and a 78 Mbps multitone orthogonal frequency-division multiple access (OFDMA) signal. The power budget of this configuration supports a 4-element antenna array.
\end{abstract}

Index Terms-Beamforming, mobile communications, optical access networks, radio over fiber (RoF) networks, WiMAX.

\section{INTRODUCTION}

$\mathbf{T}$ HE explosion of Internet traffic will be the key driver for the deployment of next-generation high-speed fixed and mobile data access networks [1]. We are going to see the massive introduction of the passive optical network (PON) and eventually its long-reach and wavelength-division multiplexed variants, with increased range and user data rates upward of $10 \mathrm{~Gb} / \mathrm{s}$ [2], [3]. Convergence of optical and wireless access networks is required in order to provide seamless connectivity to mobile users. Additionally, next-generation wireless access networks should provide mobile connections to fast-moving users at much higher data rates than currently available. This would realistically be achieved with multiple-antenna transceivers and statistical signal processing [4]. Such technologies are being integrated into newer radiofrequency (RF) communications systems, including the worldwide interoperability for microwave access (WiMAX) (IEEE 802.16d [5] and

Manuscript received January 27, 2009; revised May 12, 2009 and June 12, 2009. First published June 30, 2009; current version published September 17, 2009.

K. Prince and I. Tafur Monroy are with DTU Fotonik, Technical University of Denmark, 2800 Kgs. Lyngby, Denmark (e-mail: kpri@fotonik.dtu.dk; idtm@fotonik.dtu.dk).

M. Presi, A. Chiuchiarelli, I. Cerutti, G. Contestabile, and E. Ciaramella are with the Scuola Superiore Sant'Anna, 56124 Pisa, Italy (e-mail: marco.presi@sssup.it; andrea.chiuchiarelli@cnit.it; isabella.cerutti@sssup.it; g.contestabile@sssup.it; ernesto.ciaramella@cnit.it).

Color versions of one or more of the figures in this paper are available online at http://ieeexplore.ieee.org.

Digital Object Identifier 10.1109/JLT.2009.2026491
IEEE802.16e mobile WiMAX [6]-[10]) and the IEEE 802.11n wireless LAN [11]-[13] standards.

We consider an RF communications scheme in which signaling from the central office $(\mathrm{CO})$ propagates over an access network to remote wireless access point (WAP) units that radiate wireless RF signals to mobile customer terminals. High mobile user density may be supported by reducing the coverage area of each mobile cell. Therefore, radio over fiber (RoF) access will be a key supporting technology, allowing the flexible integration of low-loss optical transport and high-density radio access networks. It also allows reduction in the complexity of remote antenna equipment thanks to the remotization of RF processing. RoF systems should therefore evolve from today's commercially available schemes, optimized for single-antenna access, to new architectures implementing multiple-antenna transceivers [14], [15] and adaptive antenna systems (AAS) [16] which adjust the launch angle of the propagating wireless RF wavefront to maximize the receiver SNR at a wireless terminal as it moves through the coverage area. Active control of the differential delay between RF signal copies launched from each element of the WAP antenna array is, therefore, required for adaptively adjusting the beam-steering angle. In this way, the RoF access system can maximize the throughput to multiple mobile terminals while maintaining high spectral efficiency. Ideally, the differential delay of the RF signal launched from each element of the antenna array should be continuously tunable, to achieve continuous beam steering throughout the cell area. Fine-tune control of RF signal true-time delay (TTD) at each antenna element is therefore a key requirement for future RoF systems.

Previous steered-beam systems used spatial sampling of the interference pattern generated by injection-locked sources [17], optical-heterodyne-based phase compensation [18], switched delay elements [19], switching matrices [20], multiwavelength lasers [21] or a combination of multiple optical sources, multiple modulators and tunable birefringent materials [22] to vary the launch angle of the RF wave. Another early approach [23] used a tunable signal wavelength at the central office (CO), and fixed dispersion between photodetectors to control the relative signal delay. Demonstration of wavelength-dependent variable TTD has also been presented [24] using an externally-modulated wideband optical source chirped Fiber Bragg grating and optical tunable filter (OTF).

We present a novel and effective RoF system providing wideband RF signal delivery to an arrayed-antenna, based on well-known commercially available components. In this 
scheme, the beam-steering angle is controlled by adjusting OTF central wavelength at the photodetecter feeding each antenna. Our scheme allows for variable tuning of signal TTD by optical means, is transmission-protocol agnostic and natively supports a wide range of current and emerging wireless standards including IEEE 802.11, and IEEE $802.16 \mathrm{~d} / \mathrm{e}$. Our scheme exploits a single, directly-modulated wideband optical SOA source, singlemode fiber (SMF) chromatic dispersion and optical filtering to implement the TTD required for the AAS functionality. The selective filtering of a broad-spectrum optical source allows us to achieve continuous delay in the electrical RF signal and reduces the impact of uncontrolled wavelength drifts: these can affect systems with tunable [23] or multiwavelength [21] sources; thus, it is inherently very stable. Our system uses only known, reliable components and is, therefore, simpler than previous proposals. It can be used to send different RF signals to each antenna element with the appropriate delay, thus natively supports space-time codes [26] or other multiple-input multiple-output (MIMO) systems [27].

We report the first known demonstration of support for multiple-antenna WiMAX and WiFi signal transmissions over optical media and demonstrate the reliable operation of our system in achieving the desired functionality while meeting or exceeding the applicable RF signal quality specifications. These results represent a natural progression from our previous report using an R-SOA with similar complex-modulation signaling [25]. Our system successfully operates across a wide range of $\mathrm{RF}$ communications frequencies, including $2.5 \mathrm{GHz}$ (using a low-frequency R-SOA). We consider downlink performance with data flow from $\mathrm{CO}$ to WAP. We evaluated both single-channel 64-QAM IEEE 802.16d WiMAX with the highest data rate of $90 \mathrm{Mbps}$ and multitone $72 \mathrm{Mbps}$ IEEE 802.16e mobile WiMAX format with an uncoded 1024-subcarrier orthogonal frequency-division multiple access (OFDMA) signal also modulated at 64-QAM.

The paper is organized as follows. Section II introduces a theoretical description of the operating principle. Section III reports the small-signal system response, indicating jitter, gain linearity, delay response and sensitivity to OTF passband full width at half maximum (FWHM). In Sections IV and V, we present the results obtained with single-carrier WiMAX transmission, and multitone orthogonal frequency-division multiple access (OFDMA) WiMAX input, respectively. Concluding remarks are then presented.

\section{THEORETICAL ANALYSIS}

As shown in Fig. 1(a), the $\mathrm{CO}$ transmitter implements an unseeded R-SOA which is directly-modulated by the message $\mathrm{RF}$ signal. This results in a broad-spectrum $(>30 \mathrm{~nm})$ modulated optical signal which is then propagated through the SMF between $\mathrm{CO}$ and wireless access point (WAP). The chromatic dispersion $(D)$ of the SMF produces a wavelength-dependent delay in the broad-spectrum optical signal. At the WAP, the signal is split and sent to each antenna, equipped with a OTF and a photodiode (PD). By tuning each OTF, the photodetected $\mathrm{RF}$ signal has, therefore, an effective variable TTD and is ready to be radiated by the antenna. The relative TTD, $\Delta t$, obtained

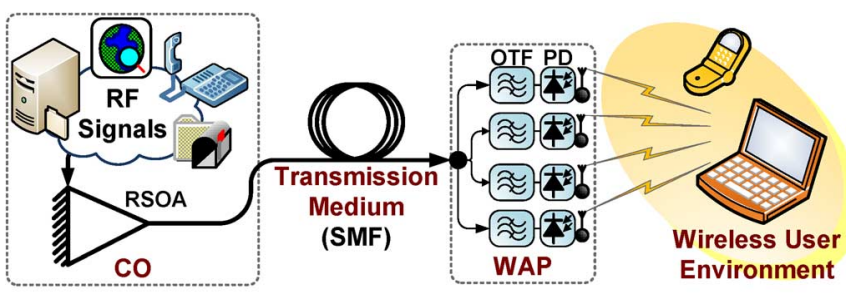

(a)

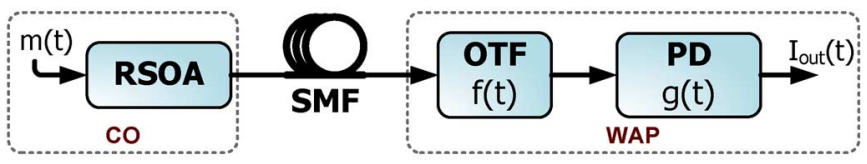

(b)

Fig. 1. (a) All-optical AAS-enabled RF over fiber scheme with directly-modulated R-SOA, dispersive transmission and OTF; and (b) block diagram.

after propagation through a SMF of length $L$, and optical filtering with wavelength detuning $\Delta \lambda$ is given by $\Delta t=D \cdot L \cdot \Delta \lambda$. We now analytically characterize the RoF signal.

\section{A. Derivation of Output Current}

Let $P_{\mathrm{ASE}}(\Omega)$ be the power spectral density of the ASE noise emitted by the R-SOA. Assuming the Optical Tuneable Filters (OTFs) have a Gaussian profile of variance $\sigma$ around the tuneable central optical angular frequency $\Omega_{0}$, the power contained in each slice is then given by

$$
P_{0}\left(\Omega_{0}\right)=\frac{1}{2 \pi} \int_{-\infty}^{\infty} P_{\mathrm{ASE}}(\Omega) e^{-\frac{\left(\Omega-\Omega_{0}\right)^{2}}{2 \sigma^{2}}} d \Omega .
$$

Assuming the ASE power spectrum to be constant over the OTF bandwidth centered at $\Omega_{0}$, (1) can be approximated to

$$
P_{0}\left(\Omega_{0}\right)=\frac{1}{2 \pi} \int_{-\infty}^{\infty} P_{\mathrm{ASE}}\left(\Omega_{0}\right) e^{-\frac{\left(\Omega-\Omega_{0}\right)^{2}}{2 \sigma^{2}}} d \Omega .
$$

As the modulating waveform is constant with respect to the scale of the coherence time of the ASE noise, the average current obtained after the photodetection is given by the following convolution product [28]

$$
\langle i(t)\rangle \approx m(t) * f(t) * g(t)
$$

where $m(t)$ is the modulating signal, $g(t)$ is the postdetection filter impulse response and $f(t)$ is the Fourier transform of the function $F(\omega)$, given by

$$
\frac{1}{2 \pi} \int_{-\infty}^{\infty} P_{\mathrm{ASE}}\left(\Omega_{0}\right) e^{-\frac{\left(\Omega-\Omega_{0}\right)^{2}}{2 \sigma^{2}}} e^{-i \omega \beta_{2} z\left(\Omega-\Omega_{0}\right)} e^{-i \omega \beta_{1} L} d \Omega .
$$

In (4), $\omega$ is the electrical baseband angular frequency, $\beta_{1}$ is the inverse of the group velocity, $\beta_{2}$ is the group velocity dispersion and $L$ is the fiber length. We remark here that $\beta_{1}$ and $\beta_{2}$ are both functions of the OTF central position $\Omega_{0}$, i.e, $\beta_{1,2}=\beta_{1,2}\left(\Omega_{0}\right)$. As probe signal, we consider a pure RF tone with modulation index $m_{0}\left(\left|m_{0}\right| \ll 1\right)$ and amplitude $A$, i.e $A\left[1+m_{0} \cos \left(\omega_{0} t\right)\right]$. With the assumption that the photodiode bandwidth is larger 
than the RF tone (such that $g(t)=\delta(t)$ ), the received current is found to be (see Section II-B)

$$
\begin{aligned}
& i(t) \propto A \sigma+A \sigma \cdot m_{0} \cos \left[\omega_{0}\left(t-\beta_{1}\left(\Omega_{0}\right) L\right)\right] \\
& \cdot \exp \left(-\frac{\left(\beta_{2} \omega_{0} L\right)^{2} \sigma^{2}}{2}\right) .
\end{aligned}
$$

To avoid heating effects, the DC term $A \sigma$ in (5) is removed by an AC coupled receiver and is, thus, neglected. From (5) it is evident that the OTF central frequency allows us to set a TTD given by $\beta_{1}\left(\Omega_{0}\right) L$ at the fiber output. Indeed at wavelengths around $1550 \mathrm{~nm}$, in a single mode fiber $\beta_{1}$ has a linear relationship versus the optical carrier. On the other hand, the OTF bandwidth $\sigma$ influences the output signal magnitude. For small $\sigma$ values the optical power delivered to each avalanche photodiode (APD) increases linearly with the OTF bandwidth. For higher OTF bandwidths, the output signal outputs decreases exponentially. Physically, this corresponds to a fading effect due to the dispersion accumulated in the wavelength-dependent delay line, which dephases the optical frequencies spread within the OTF pass band. The optimal filter bandwidth $\sigma_{\max }$ which maximizes the output RF power is then found by setting $\partial i(t) / \partial \sigma=0$, which results in

$$
\sigma_{\max }=\frac{1}{\left\|\beta_{2} \omega_{0} L\right\|} .
$$

Substitution yields an optimal OTF FWHM of $3.2 \mathrm{~nm}$.

\section{B. Detailed Derivation}

In (4), the term $e^{-i \omega \beta_{1} L}$ is constant, so it can be put outside the integration, together with $P_{\mathrm{ASE}}\left(\Omega_{0}\right)$. The expression of $F(\omega)$ is then found after the substitution $\Omega \rightarrow \Omega-\Omega_{0}$, by applying the known self-reciprocal property of Gaussian functions with respect to the Fourier transform

$$
\begin{aligned}
F(\omega) & =\frac{1}{2 \pi} P_{\mathrm{ASE}}\left(\Omega_{0}\right) e^{-i \omega \beta_{2} L} \sqrt{2 \pi} \sigma \exp \left(-\frac{\left(\beta_{2} \omega L\right)^{2} \sigma^{2}}{2}\right) \\
& =\frac{1}{\sqrt{2 \pi}} P_{\mathrm{ASE}}\left(\Omega_{0}\right) \sigma \exp \left(-\frac{\left(\beta_{2} \omega L\right)^{2} \sigma^{2}}{2}\right) e^{-i \omega \tau} \\
& =F^{\prime}(\omega) e^{-i \omega \tau} .
\end{aligned}
$$

In (7), we separated $F(\omega)$ into two parts: the odd function $F^{\prime}(\omega)$ and a constant phase delay $e^{-i \omega \tau}$. Now, with the assumption $g(t)=\delta(t)$, (3) can be calculated with the aid of the convolution theorem. In order to do this, we first write the Fourier transform of the modulating signal $M(\omega)$

$$
M(\omega)=\delta(0)+\frac{m_{0}}{2}\left[\delta\left(\omega_{0}\right)+\delta\left(-\omega_{0}\right)\right]
$$

and then evaluate the product $M \cdot F$

$$
\begin{aligned}
&\langle i(t)\rangle= \int_{-\infty}^{\infty}\left(\delta(0)+\frac{m_{0}}{2}\left[\delta\left(\omega_{0}\right)+\delta\left(-\omega_{0}\right)\right]\right) F(\omega) e^{i \omega t} d \omega \\
&=\int_{-\infty}^{\infty}\left(\delta(0)+\frac{m_{0}}{2}\left[\delta\left(\omega_{0}\right)+\delta\left(-\omega_{0}\right)\right]\right) \\
& \quad \times F^{\prime}(\omega) e^{-i \omega \tau} e^{i \omega t} d \omega \\
&=F(0)+\frac{m_{0}}{2}\left(F^{\prime}\left(\omega_{0}\right) e^{i \omega_{0}(t-\tau)}\right. \\
&\left.\quad+F^{\prime}\left(-\omega_{0}\right) e^{-i \omega_{0}(t-\tau)}\right)
\end{aligned}
$$

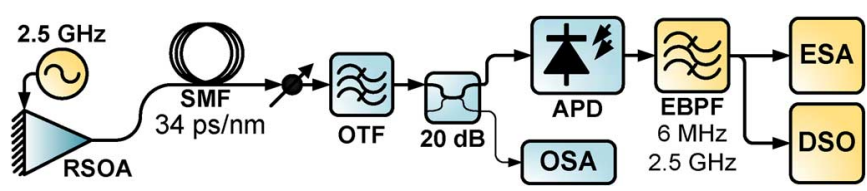

Fig. 2. Experimental setup for RF small-signal evaluation. VOA, variable optical attenuator; OTF, optical tunable filter; EBPF, electrical bandpass filter; OSA/ESA, optical/electrical spectrum analyzer; DSO, oscilloscope.

and since $F^{\prime}(\omega)$ is odd (i.e., $F^{\prime}(\omega)=F^{\prime}(-\omega)$ ), we find

$$
\langle i(t)\rangle=F(0)+m_{0} F^{\prime}\left(\omega_{0}\right) \cos \left(\omega_{0}(t-\tau)\right) .
$$

Equation (5) is then readily obtained from (10) by substituting the expression for $F^{\prime}(\omega)$. For a differential path length between the $\mathrm{CO}$ and each PD equal to an AAS element spacing of $\lambda / 2$ [29], [30], a TTD of $400 \mathrm{ps}$ would be required for $2 \pi$ steering at a frequency of $2.5 \mathrm{GHz}$.

\section{Impact of OTF FWHM on System Performance}

For fixed electrical input power to the system and small $\sigma$, the optical power delivered to each PD increases with OTF bandwidth, yielding a proportional increase in $\left|I_{\text {out }}(t)\right|$. At a fixed PD responsivity, when using large FWHM values the RF forward system gain $\left(S_{21}\right)$ depends on filter FWHM, and therefore on $\sigma$.

Arbitrarily large output RF power cannot be obtained by increasing OTF bandwidth, as fading effects become increasingly apparent at the RoF system output. The dispersion accumulated during transmission results in a time-staggered wideband optical signal reception at the PD, and the use of large FWHM OTF produces a fading in the output RF signal. Considering the wideband optical source as a synchronized group of narrowly-spaced optical wavelengths, we observe that dispersive transmission causes the PD to receive each wavelength with a small relative delay. Narrow filtering selects only a few wavelengths and their optical fields interfere constructively. Large FWHM values result in a large number of received wavelengths at the PD, each arriving with a slightly different transmission delay. The interaction between out-of-phase signal components at each wavelength results in signal fading at the PD due to the slight phase mismatch and imperfect constructive interference. Increasing the FWHM would naturally increase this fading effect.

Another constraint on FWHM selection is imposed by relative intensity noise (RIN) effects, since the system is based on the modulation of an incoherent optical source with ASE noise output and selective filtering [31]. Narrow filtering therefore increases the system output RIN due to the spontaneous emission of the optical source. OTF FWHM should, therefore, be chosen large enough to achieve a balance between RIN and fading effects, with adequate forward system gain. The optimal FWHM for a Gaussian OTF was found in (6) to be $3.2 \mathrm{~nm}$.

\section{RF SMALL-SignAL CHARACTERIZATION}

We assessed the performance of our optical TTD system using the configuration presented in Fig. 2. Our analysis was informed by the excellent systems-level treatment of RoF systems presented in [36]. The R-SOA used had a datasheet-specified electrical bandwidth of $1.25 \mathrm{GHz}$, and was 
directly intensity-modulated by a $2.5-\mathrm{GHz} \mathrm{RF}$ carrier. We used a commercially-available R-SOA providing $20 \mathrm{~dB}$ small-signal gain and $2 \mathrm{dBm}$ output saturation power when operated at $20^{\circ} \mathrm{C}$ : a temperature-independent device [32] would be preferable for a real-world deployment. The broad-spectrum optical output of the R-SOA is centered at approximately $1549 \mathrm{~nm}$, has around 20-nm FWHM, an average power of $3 \mathrm{dBm}$ and a typical ASE ripple of $0.5 \mathrm{~dB}$ at peak. The modulated broad-spectrum optical signal was transmitted through $2.1 \mathrm{~km}$ standard SMF ( $1 \mathrm{~dB}$ insertion loss and $17 \mathrm{ps} / \mathrm{nm} \cdot \mathrm{km} \cdot$ dispersion). This gave $35.7 \mathrm{ps} / \mathrm{nm}$ wavelength-dependent delay, producing $2 \pi$ beam steering at $2.5 \mathrm{GHz}$ with $11.2 \mathrm{~nm}$ OTF detuning.

The signal was passed through a variable optical attenuator (VOA), an OTF and onto an APD. This APD had $10 \mathrm{GHz}$ electro-optical bandwidth, $0.5 \mathrm{~W} / \mathrm{A}$ responsivity and an avalanche gain factor of 10 . We used a postdetector electrical band-pass filter (EBPF) with a FWHM of $6 \mathrm{MHz}$, centered at $2.5 \mathrm{GHz}$, to reject out of band noise. We evaluated system performance for various OTF having passband FWHM of 0.8, 2,3 , and $4 \mathrm{~nm}$, using spectrum analyzers (OSA, ESA) and digital storage oscilloscope (DSO).

\section{A. Small-Signal Phase Noise/Jitter}

The system was excited with a $2.5 \mathrm{GHz}$ tone at $-10 \mathrm{dBm}$ : we observed negligible intensity noise at system output, and we evaluated jitter and single-sideband (SSB) phase noise performance [33], [34]. The results of SSB phase noise $S(f)$ assessments at system input and output are shown in Fig. 3(a) for various OTF implementations. For $f_{\text {offset }}$ in the interval $\left[10,10^{6}\right] \mathrm{Hz}$, the output signal phase noise closely followed the synthesizer characteristic, with less than $10 \mathrm{~dB}$ difference between the two over this range. For offset frequency in the interval $\left(10^{3}, 3 \times 10^{3}\right) \mathrm{Hz}$, we noted an almost flat phase noise response. Between approximately $3 \mathrm{kHz}$ and $10 \mathrm{MHz}$ offset frequency, the phase noise decreased rapidly with increasing frequency offset. Above $10 \mathrm{MHz}$, the system phase noise remained below $-150 \mathrm{dBc} / \mathrm{Hz}$, which is comparable with the noise floor of the analyzer used. The postdetector RF filter accounts for the sharp roll-off observed in the phase noise characteristic for frequency offset greater than $3 \mathrm{MHz}$. The worst phase noise performance was obtained for the OTF with the highest bandwidths ( 3 and $4 \mathrm{~nm}$ ), and the best results were obtained with $2 \mathrm{~nm}$ FWHM.

The integrated SSB jitter $\delta_{t}$ of a signal within frequency interval $\left[f_{\min }, f_{\max }\right]$ is given by [34]

$$
\delta_{t}=\frac{1}{2 \pi f_{c}} \sqrt{2 \int_{f_{\min }}^{f_{\max }} S(f) d f} .
$$

We experimentally evaluated system input and output SSB jitter, from a minimum offset frequency $\left(f_{\min }\right)$ of $100 \mathrm{~Hz}$, up to $f_{\max }$ of $20 \mathrm{GHz}$, and present the results in Fig. 3(b). The selected $f_{\min }$ value ensured that the tails of the carrier signal would not be included in the calculation of the phase noise of the system, due to frequency resolution limitations of the ESA [33]. The best resolution of the device used was $316 \mathrm{~Hz}$. From Fig. 3(b), we observed that all OTF implementations provide less than $1.5 \mathrm{ps}$ integrated SSB jitter in the frequency interval $\left[10^{3}, 2 \times 10^{6}\right] \mathrm{Hz}$ :

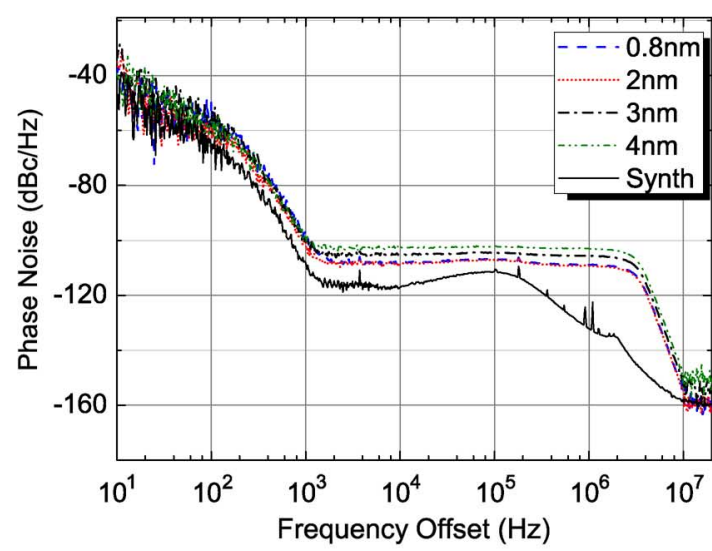

(a)

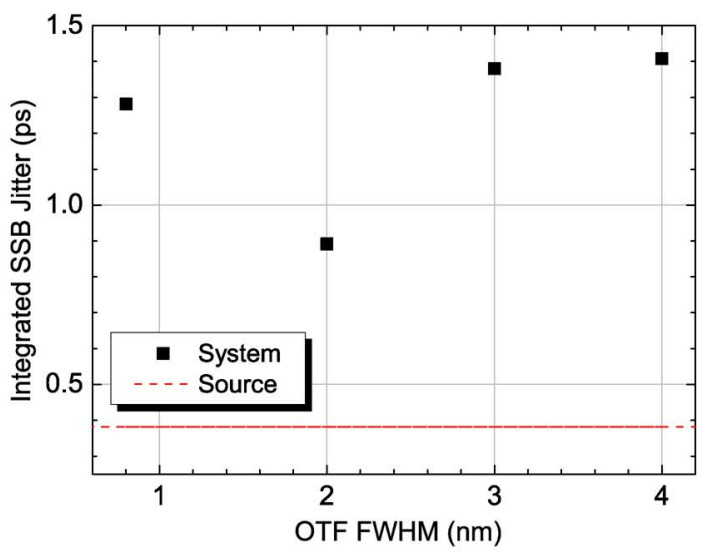

(b)

Fig. 3. (a) Phase noise; (b) jitter versus FWHM in the interval $\left[10^{3}, 2 \times\right.$ $\left.10^{6}\right] \mathrm{Hz}$.

best jitter results were obtained with the $2 \mathrm{~nm}$ OTF. We assessed the carrier-to-noise ratio (CNR) for the output signal measured at $1 \mathrm{kHz}$ offset frequency; all OTF produced CNR better than $-100 \mathrm{dBc} / \mathrm{Hz}$.

\section{B. RF Gain Characteristics}

As the best jitter and phase noise results were observed with the $2 \mathrm{~nm}$ OTF, we used this filter and assessed system linearity as a function of the input RF power [35]. Without the postdetector EBPF, we observed linear system response for source power levels between $-30 \mathrm{dBm}$ and $+10 \mathrm{dBm}$. Inclusion of the postdetection RF bandpass-filter produced a shaped gain spectrum. We also observed that system gain was almost constant at $-15 \mathrm{~dB}$ for input power below approximately $-10 \mathrm{dBm}$, which is comparable with the literature [36]. Above this threshold, system gain begins to decrease steadily with increasing input power, resulting in loss of linearity. These results are presented in Fig. 4; similar trends were obtained for other OTF units evaluated.

\section{System Linearity}

We evaluated system-induced distortion by assessing second and third-order intermodulation according to [37] using two unmodulated carriers of equal magnitude separated by $200 \mathrm{kHz}$. We used the setup of Fig. 2, with 2 nm OTF; results were obtained at a resolution bandwidth of $316 \mathrm{~Hz}$, the noise floor was at 


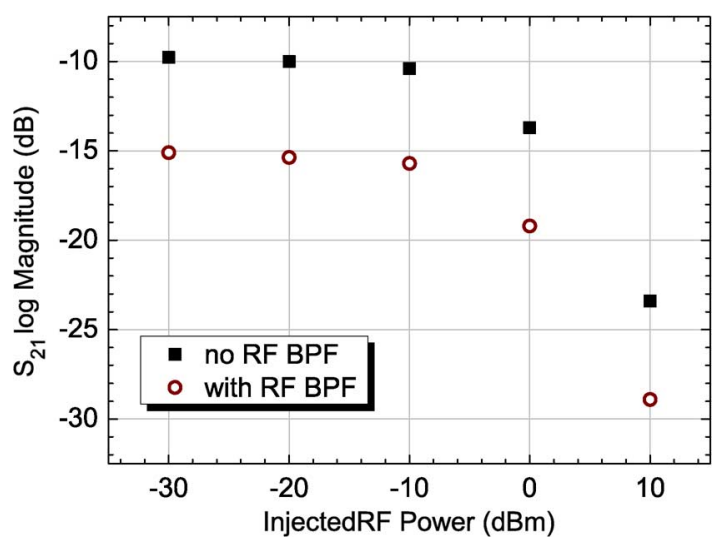

Fig. 4. System magnitude response $\left(S_{21}\right)$ versus input RF power for $2 \mathrm{~nm}$ OTF.

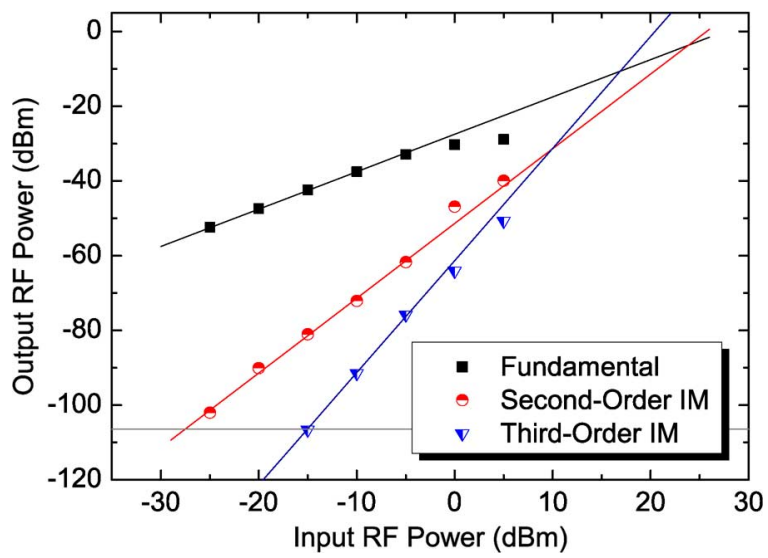

Fig. 5. Two-tone fundamental and intermodulation product (second- \& thirdorder) output power as a function of input RF signal power. Dynamic range, $\mathrm{IMF}_{3}=65 \mathrm{~dB} \mathrm{~Hz}^{2 / 3}$ : tone separation, $200 \mathrm{kHz}$; meas. bandwidth, $316 \mathrm{~Hz}$.

$-106 \mathrm{dBm}$. The evolution of output fundamental and intermodulation (IM) terms with input RF signal power is shown in Fig. 5. The second-order free dynamic range $\left(\mathrm{IMF}_{2}\right)$ is $52 \mathrm{~dB} \mathrm{~Hz}^{2 / 3}$ and the third-order free dynamic range $\left(\mathrm{IMF}_{3}\right)$, also called the spurious-free dynamic range (SFDR), is $65 \mathrm{~dB} \mathrm{~Hz}^{2 / 3}$. This is comparable with the values reported in [38] (for an amplified $120 \mathrm{~km}$ optical link operating at $5 \mathrm{GHz}$, with an EAM source), but less than the $106 \mathrm{~dB} \mathrm{~Hz}^{2 / 3}$ obtained using a DBR source without RF preamplification [39], or the $115 \mathrm{~dB} \mathrm{~Hz}^{2 / 3}$ obtained using a DFB with RF preamplification [40]. The $1 \mathrm{~dB}$ compression point of our system was at $-4 \mathrm{dBm}$ input power, which is suitable for short-range picocellular applications requiring low launch RF power levels: other authors [20] have previously reported $+7 \mathrm{dBm}$ output power level corresponding to a $1 \mathrm{~dB}$ compression point, but with a more complex architecture. We observed the second-order intercept point at an input power of $+25 \mathrm{dBm}$ and a third-order distortion intercept at $+16 \mathrm{dBm}$.

\section{Sensitivity to OTF Central Wavelength and Bandwidth}

We assessed the variation of TTD with filter detuning across the operating band. This is reported in Fig. 6(a). The TTD was independent of OTF FWHM; however, the RF output power varied with FWHM: the maximum output RF signal was observed for the $2 \mathrm{~nm}$-filter and the output RF power decreased at higher OTF bandwidth as also anticipated by (5). At any given

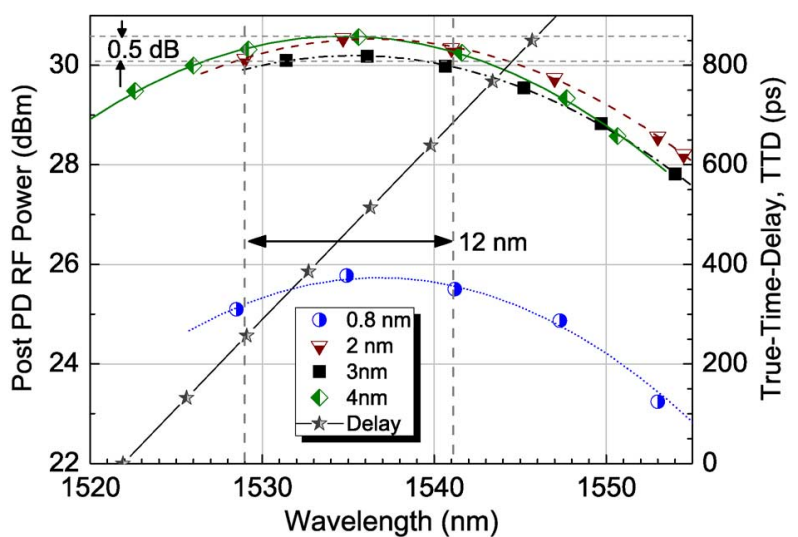

(a)

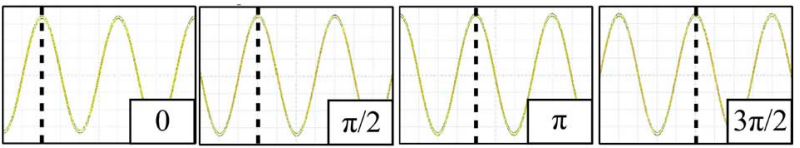

(b)

Fig. 6. (a) Output RF power and TTD versus detuning with various OTF for 2.5-GHz signal; (b) output signal at various TTD (across 12-nm detuning), with 2-nm OTF. (Vert. scale: $10 \mathrm{mV} /$ div., power variation within $0.5 \mathrm{~dB}$ ).

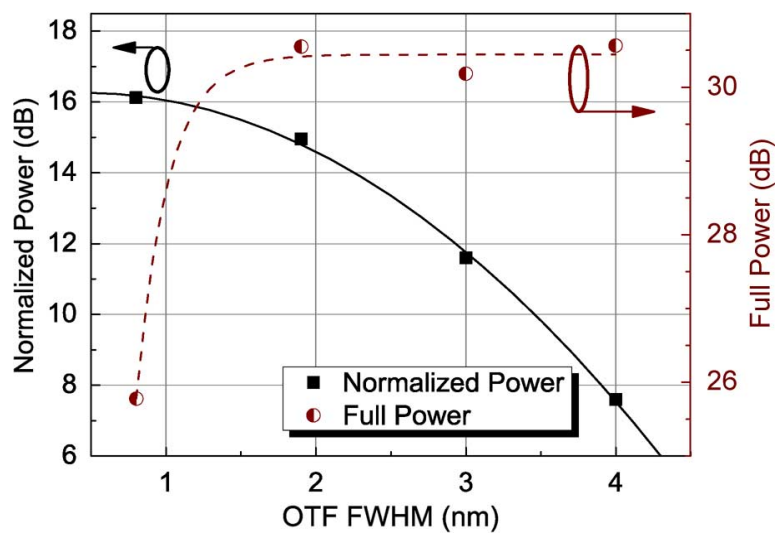

Fig. 7. Normalized system gain as a function of OTF FWHM; APD input power fixed at $-30 \mathrm{dBm}$ (solid), full optical power onto the APD (dashed).

wavelength, all filter implementations produced output power profiles proportional to the ASE noise power spectral density (PSD) at that wavelength. The detuning range of $12 \mathrm{~nm}$ (for full $2 \pi$ beam steering) had an associated power excursion within $1.2 \mathrm{~dB}$; samples of the output signal amplitude for various TTD are presented in Fig. 6(b). System response was then evaluated for OTF implementations with varying FWHM. As the filters that we used had unequal insertion losses and unequal passband characteristics (Gaussian OTF were not available for all FWHM values evaluated), measurements were taken with maximum available optical power into the APD (i.e., with zero VOA attenuation), and also with VOA adjusted to maintain a constant $-30 \mathrm{~dB}$ input average optical power into the APD: the results are presented in Fig. 7. For the case with controlled optical input power to the APD (solid line), we observed that the RF output power decreased with increasing OTF FWHM. We concluded that this test scenario isolated the RF fading phenomenon (by removing the gain associated with increased APD input power) 


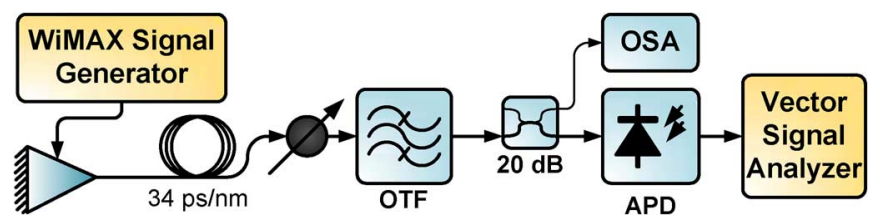

Fig. 8. System layout for WiMAX evaluation.

that was predicted in (5) and we clearly see that the output electrical RF signal is diminished with increased FWHM, and that the observations closely follow the trace predicted by theory (which is parabolic for a log scale). When maximum optical power was sent to the APD (dashed line), the output RF power was approximately $26 \mathrm{dBm}$ for the $0.8 \mathrm{~nm}$ OTF; all other OTF units provided approximately $30.5 \mathrm{dBm}$. We believe that the discrepancy in OTF insertion loss and passband characteristic mask the trends predicted by (5), although there is a marked decrease in output power at low FWHM. Theory indicates a similar decrease in output RF power if the FWHM were further increased, but we were unable to evaluate these conditions.

\section{Single-CARRIER WIMAX SignAL TRANSMISSION}

We evaluated system performance with single-carrier WiMAX IEEE 802.16d signals: the MAN SCa PHY [5] regulates single-carrier transmissions at frequencies below $11 \mathrm{GHz}: 120 \mathrm{~km}$ AAS support is optional within the standard. It supports 16- and 64-QAM, allowing FDD and/or TDD with TDM(A) downlink and TDMA uplink, with a maximum allowable frame duration of $20 \mathrm{~ms}$. The maximum allowable error vector magnitude (EVM) for 64-QAM signaling is $3.1 \%$ and the symbol-to-symbol timing jitter must be within $2 \%$ : power control is required, in $1 \mathrm{~dB}$ increments, although maximum transmit power levels are set by local authorities and there are no intermodulation specifications in the standard.

The test signal was obtained from an Agilent E4438C ESG generator. System EVM performance was characterized with a 26.5 GHz Agilent N9020A MXA signal analyzer. Our analysis was done for 64-QAM, which is the fastest modulation scheme required by the WiMAX standard. The layout is shown in Fig. 8. We used a carrier frequency of $2.4 \mathrm{GHz}$ and symbol rate of $15 \mathrm{Msps}$ (million symbols per second), equivalent to $90 \mathrm{Mbps}$. At this carrier frequency, $(\lambda / 2=6.25 \mathrm{~cm})$ antenna spacing required 417 ps TTD for $2 \pi$ steering, this was achieved with $11.7 \mathrm{~nm}$ OTF detuning.

A sample of the received signal constellation is presented in Fig. 9(a); Fig. 9(b) presents EVM as a function of drive electrical RF power. RF power in the range $[-6,-14] \mathrm{dBm}$ provided acceptable EVM: we note from Fig. 4 that this overlaps with the power required for good system linearity. We also assessed EVM variation with received optical power (at fixed wavelength and input RF power) and present the results in Fig. 9(c): we observed approximately $6 \mathrm{~dB}$ system margin to the EVM threshold. Observations of the variation of RMS EVM with OTF detuning is presented in Fig. 9(d), indicating $12 \mathrm{~nm}$ detuning associated with less than $0.2 \%$ EVM excursion: acceptable EVM was obtained across the entire operating range of the OTF.

Low EVM and output power variation with OTF detuning and the optical power margin, indicate the feasiblity of optical split

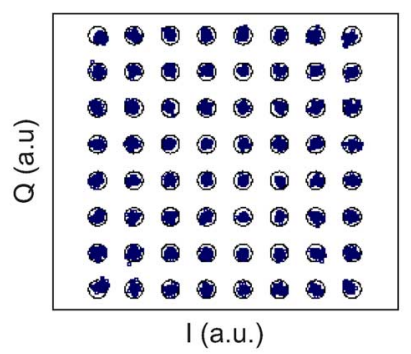

(a)

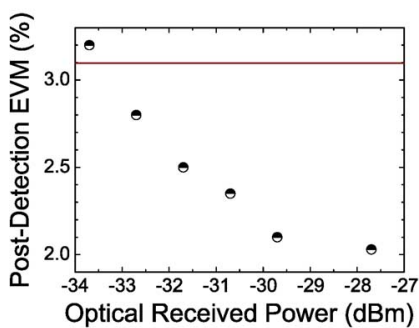

(c) (b)

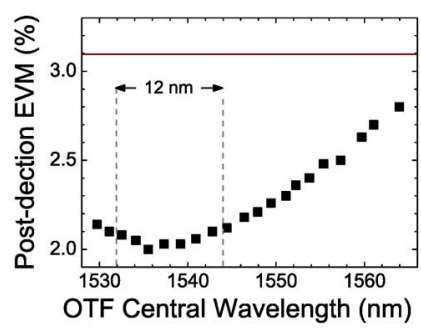

(d)

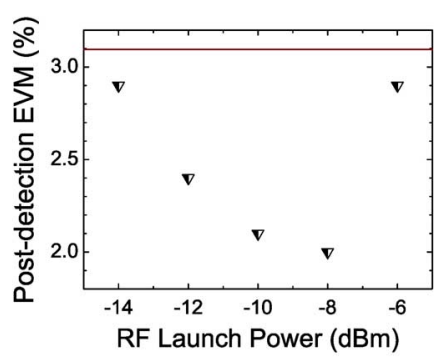

Fig. 9. Single-carrier 64-QAM WiMAX signal at $90 \mathrm{Mbps}$, (a) constellation; and output signal EVM as a function of (b) input RF power, (c) APD power, (d) OTF wavelength. 3.1\% IEEE 802.16d WiMAX EVM threshold indicated.

and filtering at the WAP to implement a quad-antenna array configuration with low-loss splices: differential OTF wavelength selection would control the TTD delay at each antenna element and, hence, the angle of the launched RF wave. We then investigated system performance with a multicarrier OFDMA payload.

\section{Multitone WIMAX SignAl TRANSMission}

IEEE 802.16e Mobile WiMAX [7] is heavily favored to become a dominant technology for supporting wireless data access services. It will require multiple transceiver antennas at WAP nodes for integrated AAS support: the MAN-OFDMA PHY [6] defines the air interface requirements. OFDMA signaling is implemented with up to 2048 subcarriers: for subscriber station and base station (or WAP), the relative constellation error (RCE) with an encoded (rate 3/4) 64-QAM transmission must be less than $-30 \mathrm{~dB}$. Maximum transmit power levels and intermodulation performance specifications are not mandated.

We used the setup shown in Fig. 8 to evaluate the transmission of mobile WiMAX OFDMA signals. Performance was assessed using the relative constellation error (RCE) obtained on the uncoded data subchannels, as defined in [6]. The generated OFDMA signal allowed a selection between a partial use of subchannels (PUSC) or a full use of subchannels (FUSC) format: we opted for PUSC and fixed 64-QAM modulation on all data subcarriers. We evaluated time-division multiplex system using a ratio of downlink/total frame duration $\left(T_{\mathrm{DL}} / T_{\mathrm{TOTAL}}\right)$ of $99 \%$ and $50 \%$. The test OFDMA signal had the following parameters: 2048 subcarriers, $20 \mathrm{MHz}$ signal bandwidth, no FEC coding, $5 \mathrm{~ms}$ frame duration, 1/4 guard interval and a central frequency of $2.5 \mathrm{GHz}$. The maximum data throughput was $72.3 \mathrm{Mbit} / \mathrm{s}$. Since R-SOA typically have a highly non-linear electro-optical transfer function, which is unsuitable for OFDMA signals, we optimized R-SOA bias-point and input RF power to operate the $\mathrm{R}-\mathrm{SOA}$ in the quasi-linear region, in order to reduce intermodulation distortion between sub-carriers and obtain adequate SNR. 


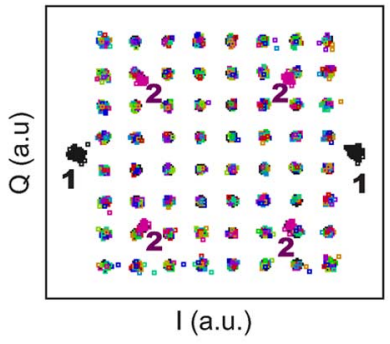

(a)

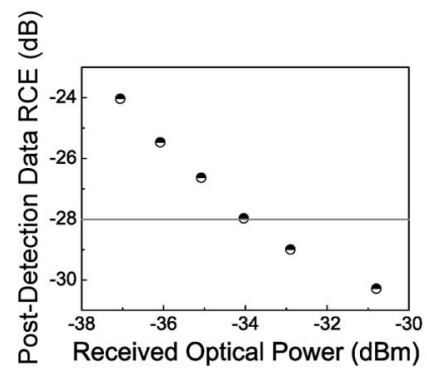

(c)

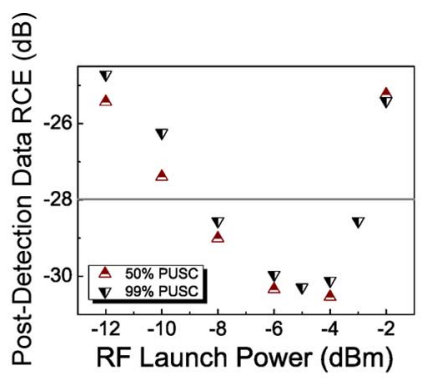

(b)

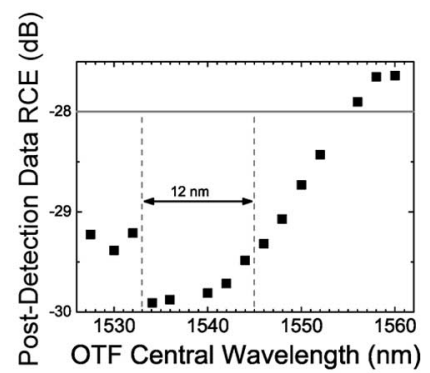

(d)
Fig. 10. 64-QAM, 2048-subcarrier, 99\% downlink OFDMA IEEE 802.16e WiMAX frame, (a) constellation, BPSK pilot (1) and QPSK preamble (2): data RCE variation with (b) launch RF power, (c) received APD power and (d) OTF wavelength. RCE below -28-dB WiMAX threshold for 64-QAM.

Fig. 10(a) illustrates the constellation of the signal obtained at the APD output. It is possible to clearly distinguish between the BPSK pilots, the QPSK used in the packet preamble and the 64-QAM used in each burst of the WiMAX signal [6]. Fig. 10(b) shows the performance of OFDMA PUSC signals having 50\% and a $99 \%$ downlink sub-frame duration. In both cases, the optimal RF driving power was approximately $-5 \mathrm{dBm}$. The $99 \%$ represented more stringent requirements, so all subsequent evaluations were taken with this setting. The IEEE 802.16e RCE requirement of $-28 \mathrm{~dB}$ for a 64-QAM coded (rate 3/4) signal was met for RF driving power levels in the range $[-9,-3] \mathrm{dBm}$. Fig. 10(c) reports the system performance in terms of received optical power. The sensitivity (measured at $-28 \mathrm{~dB} R C E$ ) determines the maximum number of antennas (i.e., the splitting ratio) supported by this system. It matches the IEEE $802.16 \mathrm{e}$ requirements when the received optical power level exceeds $-34 \mathrm{dBm}$. In our case, modulated ASE experienced a 1-dB loss in the feeder and $22 \mathrm{~dB}$ at the OTF ( $20 \mathrm{~dB}$ due to OTF rejection). RCE variation with OTF detuning is reported in Fig. 10(d): IEEE 802.16 e requirements are met in a detuning range wider than the $12 \mathrm{~nm}$ required for full beam steering: this range is associated with less than $1 \mathrm{~dB}$ RCE penalty. Due to the low power and RCE penalty with OTF detuning, and $3 \mathrm{~dB}$ optical power margin, a dual-antenna WAP is feasible for mobile WiMAX-compliant signaling: a higher output power device may allow additional antennas.

\section{CONCLUSION}

We presented a radio-over-fiber system suitable for adaptive antenna systems based on spectrum-slicing of a directly-modulated R-SOA. We have successfully characterized the system, and evaluated it with single-tone and OFDMA WiMAX signals. This optical source can support four antennas in the single-tone implementation, and the use of an electrical preamplifier allows the transmission of an optical signal that can be distributed to two antenna elements. We note that operation with optimal input RF power levels implies low RF electrical power at the output of each photodiode driving the antenna array: a higher-powered optical source or low-noise RF amplification at the WAP could improve system gain while preserving the other advantages. In all cases, standard-compliant operation was obtained with the maximum modulation depth specified (64-QAM). The OTF tuning required to achieve full beam steering may safely be done with the delayed output still meeting performance criteria. Sample constellation diagrams demonstrated good signal transmission through our optical system.

\section{ACKNOWLEDGMENT}

The authors would like to thank M. Pagano and R. Sacchi of Agilent Technologies for technical support and the loan of the N9020A MXA signal analyzer and E4438C ESG generator.

\section{REFERENCES}

[1] S. Cherry, "Edholm's law of bandwidth," IEEE Spectrum, vol. 41, no. 7, pp. 58-60, Jul. 2004.

[2] K. Grobe and J.-P. Elbers, "PON in adolescence: From TDMA to WDM-PON," IEEE Commun. Mag., vol. 46, no. 1, pp. 26-34, Jan. 2008.

[3] C.-H. Lee, S.-M. Lee, K.-M. Choi, J.-H. Moon, S.-G. Mun, K.-T. Jeong, J. H. Kim, and B. Kim, "WDM-PON experiences in Korea (invited)," J. Opt. Netw., vol. 6, no. 5, pp. 451-464, 2007.

[4] W. Konhäuser, "Broadband wireless access solutions-Progressive challenges and potential value of next generation mobile networks," Wireless Pers. Commun., vol. 37, no. 3-4, pp. 243-259, 2006.

[5] IEEE LAN/MAN Standards Cttee., IEEE Std.802.16-2004, Oct. 2004.

[6] IEEE LAN/MAN Standards Cttee., IEEE Std. 802.16e-2005, Feb. 2006.

[7] K. H. Teo, Z. Tao, and J. Zhang, "The mobile broadband WiMAX standard [standards in a nutshell]," IEEE Signal Process. Mag., vol. 24, no. 5, pp. 144-148, Sep. 2007.

[8] S. Cherry, "South Korea pushes mobile broadband," IEEE Spectrum, vol. 42, no. 9, pp. 14-16, Sep. 2005.

[9] C. Nam, S. Kim, and H. Lee, "The role of WiBro: Filling the gaps in mobile broadband technologies," Technolog. Forecast. Social Change, vol. 75, no. 3, pp. 438-448, Mar. 2008.

[10] S. J. Vaughan-Nichols, "Mobile WiMAX: The next wireless battle ground," Computer, vol. 41, no. 6, pp. 16-18, 2008.

[11] Unapproved draft standard, IEEE P802.11n/D4.00, Mar. 2008.

[12] R. Nee, V. K. Jones, G. Awater, A. Zelst, J. Gardner, and G. Steele, "The 802.11n MIMO-OFDM standard for wireless LAN and beyond," Wireless Pers. Commun., vol. 37, no. 3-4, pp. 445-453, 2006.

[13] Y. Xiao, "IEEE 802.11n: Enhancements for higher throughput in wireless LANs," IEEE Wireless Commun., vol. 12, no. 6, pp. 82-91, Dec. 2005.

[14] S. Alamouti, "A simple transmit diversity technique for wireless communications," IEEE J. Sel. Areas Commun., vol. 16, no. 8, pp. 1451-1458, Aug. 1998.

[15] V. Tarokh and H. Jafarkhani, "A differential detection scheme for transmit diversity," IEEE J. S. Ar. Cm., vol. 18, no. 7, pp. 1169-1174, 2000

[16] J. Winters, "Smart antennas for wireless systems," IEEE Pers. Commun., vol. 5, no. 1, pp. 23-27, Feb. 1998.

[17] M. Tamburrini, M. Parent, L. Goldberg, and D. Stillwell, "Optical feed for a phased array microwave antenna," Electron. Lett., vol. 23, no. 13, pp. 680-681, June 1987.

[18] E. Toughlian and H. Zmuda, "A photonic variable RF delay line for phased array antennas," J. Lightw. Tech., vol. 8, no. 12, pp. 1824-1828, Dec. 1990. 
[19] W. Ng, A. Walson, G. Tangonan, J. Lee, and I. Newberg, "Optical steering of dual band microwave phased array antenna using semiconductor laser switching," Electron. Lett., vol. 26, no. 12, pp. 791-793, June 1990

[20] M. Piqueras, G. Grosskopf, B. Vidal, J. Herrera, J. Martinez, P. Sanchis, V. Polo, J. Corral, A. Marceaux, J. Galiere, J. Lopez, A. Enard, J.-L. Valard, O. Parillaud, E. Estebe, N. Vodjdani, M.-S. Choi, J. den Besten, F. Soares, M. Smit, and J. Marti, "Optically beamformed beamswitched adaptive antennas for fixed and mobile broad-band wireless access networks," IEEE Trans. Microw. Theory. Tech., vol. 54, no. 2, pp. 887-899, Feb. 2006.

[21] B. Vidal, D. Madrid, J. Coffal, and J. Marti, "Novel photonic true-timedelay beamformer based on the free-spectral-range periodicity of arrayed waveguide gratings and fiber dispersion," IEEE Photon. Technol. Lett., vol. 14, no. 11, pp. 1614-1616, Nov. 2002.

[22] L. Jofre, C. Stoltidou, S. Blanch, T. Mengual, B. Vidal, J. Marti, I. McKenzie, and J. M. del Cura, "Optically beamformed wideband array performance," IEEE Trans. Antennas Propagat., vol. 56, no. 6, pp. 1594-1604, Jun. 2008.

[23] R. Esman, M. Frankel, J. Dexter, L. Goldberg, M. Parent, D. Stilwell, and D. Cooper, "Fiber-optic prism true time-delay antenna feed," IEEE Photon. Technol. Lett., vol. 5, no. 11, pp. 1347-1349, Nov. 1993.

[24] B. Zhou, X. Zheng, X. Yu, H. Zhang, Y. Guo, and B. Zhou, "Optical beamforming networks based on broadband optical source and chirped fiber grating," IEEE Photon. Technol. Lett., vol. 20, no. 9, pp. 733-735, Sep. 2008

[25] K. Prince, A. Chiuchiarelli, M. Presi, I. T. Monroy, and E. Ciaramella, "All-optical delay technique for supporting multiple antennas in a hybrid optical-Wireless transmission system," presented at the 21st Meet. IEEE Lasers Electro-Optics Society, Newport Beach, CA, Nov. 2008.

[26] D. Gesbert, M. Shafi, D.-S. Shiu, P. Smith, and A. Naguib, "From theory to practice: An overview of MIMO space-time coded wireless systems," IEEE J. Sel. Areas Commun., vol. 21, no. 3, pp. 281-302, Apr. 2003.

[27] A. Paulraj, D. Gore, R. Nabar, and H. Bölcskei, "An overview of MIMO communications-A key to gigabit wireless," Proc. IEEE, vol. 92, no. 2, pp. 198-218, Feb. 2004.

[28] G. Pendock and D. Sampson, "Transmission performance of high bit rate spectrum-sliced WDM systems," J. Lightw. Technol., vol. 14, no. 10, pp. 2141-2148, Oct. 1996.

[29] G. Foschini and M. Gans, "On limits of wireless communications in a fading environment when using multiple antennas," Wireless Personal Commun., vol. 6, no. 3, pp. 311-335, Mar. 1998.

[30] M. Stoytchev, H. Safar, A. Moustakas, and S. Simon, "Compact antenna arrays for MIMO applications," in Proc. IEEE Antennas and Propagation Society Int. Symp., 2001, vol. 3, pp. 708-711.

[31] A. D. McCoy, B. C. Thomsen, M. Ibsen, and D. J. Richardson, "Experimental study on receiver filtering effects in a spectrum-sliced incoherent light WDM system using SOA based noise reduction," in Proc. ECOC, Rimini, Italy, Sep. 2003, pp. 866-867, WE4P156.

[32] K. Y. Cho, Y. Takushima, K. R. Oh, and Y. C. Chung, "Operating wavelength range of $1.25-\mathrm{Gb} / \mathrm{s}$ WDM PON implemented by using uncooled RSOA's," presented at the OFC/NFOEC, San Diego, CA, 2008, oTuH3, unpublished.

[33] L. K. Oxenløwe, "Optical Signal Processing With Semiconductor Components," Ph.D. dissertation, Tech. Univ. Denmark, Kgs. Lyngby, Denmark, 2002.

[34] J. Hargreaves, P. Juodawlkis, J. Plant, J. Donnelly, J. Twichell, F. Rana, and R. Ram, "Residual phase-noise measurements of actively mode-locked fiber and semiconductor lasers," in Proc. LEOS, 2001, pp. 115-116.

[35] C. H. Cox, III, H. Roussell, R. Ram, and R. Helkey, "Broadband, directly modulated analog fiber link with positive intrinsic gain and reduced noise figure," in Proc. Int. Topical Mtg. on Microw. Phot., 1998, pp. 157-160.

[36] C. H. Cox, III, E. I. Ackerman, G. E. Betts, and J. E. Prince, "Limits on the performance of RF-over-fiber links and their impact on device design," IEEE Trans. Microw. Theory Tech., vol. 54, no. 2, pp. 906-920, Feb. 2006

[37] C. H. Cox, III, Analog Optical Links: Theory and Practice. Cambridge, U.K.: Cambridge Univ. Press, 2004, ch. 6.2.2, pp. 205-217.
[38] H.-H. Lu, S.-J. Tzeng, and Y.-L. Liu, "Intermodulation distortion suppression in a full-duplex radio-on-fiber ring network," IEEE Photon. Technol. Lett., vol. 16, no. 2, pp. 602-604, Feb. 2004.

[39] L. A. Johansson, Y. A. Akulova, C. Coldren, and L. A. Coldren, "Improving the performance of sampled-grating DBR laser-based analog optical transmitters," J. Lightw. Technol., vol. 26, no. 7, pp. 807-815, Jul. 2008

[40] A. Razibul Islam, G. Town, and A. Tariqul Islam, "PAPR and SFDR of an OFDM/RoF link," in Proc. OECC/ACOFT, Jul. 2008, pp. 1-2.

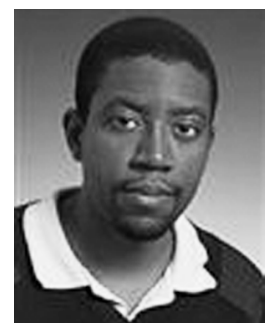

Kamau Prince received the B.Sc. degree (first class honours) in electrical engineering from the University of the West Indies, St. Augustine, Trinidad, in 1999, and the Graduate Diploma in telecommunications engineering and the M.Eng.Sci. degree from the University of Melbourne, Victoria, Australia, in 2003 and 2006, respectively. He is currently pursuing the $\mathrm{Ph} . \mathrm{D}$. degree in optical communications at DTU Fotonik, Technical University of Denmark.

Marco Presi received the Laurea degree in physics from "La Sapienza" University of Rome, Italy, in 2001, and the Ph.D. degree in applied physics from the University of Pisa, Italy, in 2006.

Since 2007, he has been a Research Associate at the Scuola Superiore Sant'Anna, Pisa, Italy, where he currently works on WDM optical systems. He has coauthored more than 50 papers published in international peer-reviewed journals and in international conferences.

Andrea Chiuchiarelli received the Laurea degree in electronic engineering from the University of L'Aquila, Italy, in March 2005. He is currently pursuing the Ph.D. degree at the Scuola Superiore Sant'Anna in the Optical Systems group of CEIIC (Center of Excellence for Information and Communication Engineering), Pisa, Italy.

From November 2005 to November 2006, he was with the Inter-University National Consortium for Telecommunications (CNIT).

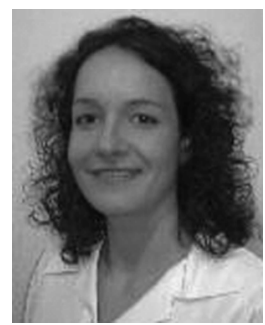

Isabella Cerutti received the "Laurea" degree in electrical engineering from the Politecnico di Torino, Italy, 1998, and the Ph.D. degree from the University of Texas at Dallas (UTD) in 2002.

From 2002 to 2006, she was a postdoctorate research associate at UTD and then at the Scuola Superiore Sant'Anna (SSSUP), Italy. Since 2007, she has been an Assistant Professor at SSSUP. She coauthored more than 50 publications in international journals and conference proceedings.

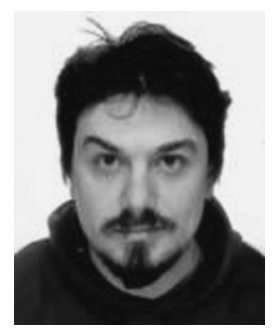

Giampiero Contestabile received the Laurea degree in physics from "La Sapienza" University of Rome, Italy, in 1998, and the Ph.D. degree in electrical engineering-telecommunications from "Tor Vergata" University of Rome, Italy, in 2001.

Between 1996 and 2000, he was with the Semiconductor Devices Group of "Fondazione Ugo Bordoni", Rome. In 2001, he was with Optospeed Italia. Since September 2002, he has been an Assistant Professor at the "Scuola Superiore Sant'Anna" University of Pisa, Italy. He has coauthored more than 90 papers published in international peer-reviewed journals and presented at leading international conferences. His main research interests are advanced WDM systems, optical packet switched networks, access networks and applications of semiconductor optical amplifiers.

Dr. Contestabile is one of the reviewers of the Journal OF LightwaVe TeChNology, Photonics Technology Letters, OpticsExpress, Optics Letters, and Optics Communications. 
Idelfonso Tafur Monroy received the M.Sc. degree in multichannel telecommunications from the Bonch-Bruevitch Institute of Communications, St. Petersburg, Russia, in 1992, the Technology Licenciate degree in telecommunications theory from the Royal Institute of Technology, Stockholm, Sweden, and the $\mathrm{Ph} . \mathrm{D}$. degree from the Electrical Engineering Department, Eindhoven University of Technology, The Netherlands, in 1999.

$\mathrm{He}$ is currently Head of the metro-access and short range communications group of the Department of Photonics Engineering, Technical University of Denmark. He was an Assistant Professor until 2006 at the Eindhoven University of Technology. Currently, he is an Associate Professor at the Technical University of Denmark. He has participated in several European research projects, including the ACTS, FP6, and FP7 frameworks (APEX, STOLAS, LSAGNE, MUFINS). At the moment, he is in involved the ICT European projects GiGaWaM, ALPHA, BONE, and EURO-FOS. His research interests are in hybrid optical-wireless communication systems, coherent detection technologies and digital signal processing receivers for baseband and radio-over-fiber links, optical switching, nanophotonic technologies, and systems for integrated metro and access networks, short range optical links, and communication theory.

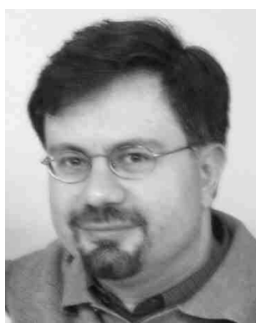

Ernesto Ciaramella (M'06) was born in Rome, Italy, in 1967. He received the Laurea degree (cum laude) from La Sapienza University, Rome, Italy, in 1991.

In 1992, he was awarded a scholarship by Alcatel. During 1992-1994, he was with Fondazione Ugo Bordoni, working on nonlinear optical effects. From 1994-1998, he was with CSELT, Turin, Italy, concerned with linear and nonlinear propagation effects in optical fibers and with system modeling. From 1998-2000, he was with the Fondazione Ugo Bordoni, working on optical transmission systems and network architectures. In 2001-2002, he was a Research Manager at CNIT, Pisa, Italy. Since 2002, he has been an Associate Professor at the Scuola Superiore Sant'Anna, Pisa, Italy. His present research activity covers optical transmission systems, nonlinear effects, fiber transmission impairments, all-optical processing, and optical packet switching. He has participated in various European research projects, published more than 100 papers, and is the author/coauthor of ten international patents. He is presently serving on the Technical Program Committee of the Optical Fiber Communication Conference (OFC). 